\title{
First measurement and correction of nonlinear errors in the experimental insertions of the CERN Large Hadron Collider
}

\author{
E. H. Maclean \\ CERN, CH-1211 Geneva, Switzerland; Cocroft Institute, Daresbury WA4 4AD, \\ United Kingdom; University of Manchester, Manchester M13 9PL, United Kingdom \\ R. Tomás, M. Giovannozzi, and T. H. B. Persson \\ CERN, CH-1211 Geneva, Switzerland
}

(Received 17 December 2014; published 7 December 2015)

\begin{abstract}
Nonlinear magnetic errors in low- $\beta$ insertions can contribute significantly to detuning with amplitude, linear and nonlinear chromaticity, and lead to degradation of dynamic aperture and beam lifetime. As such, the correction of nonlinear errors in the experimental insertions of colliders can be of critical significance for successful operation. This is expected to be of particular relevance to the LHC's second run and its high luminosity upgrade, as well as to future colliders such as the Future Circular Collider. Current correction strategies envisioned for these colliders assume it will be possible to calculate optimized local corrections through the insertions, using a magnetic model of the errors. This paper shows however, that reliance purely upon magnetic measurements of the nonlinear errors of insertion elements is insufficient to guarantee a good correction quality in the relevant low- $\beta^{*}$ regime. It is possible to perform beam-based examination of nonlinear magnetic errors via the feed-down to readily observed beam properties upon application of closed orbit bumps, and methods based upon feed-down to tune have been utilized at RHIC, SIS18, and SPS. This paper demonstrates the extension of such methodology to include direct observation of feed-down to linear coupling in the LHC. It is further shown that such beam-based studies can be used to complement magnetic measurements performed during LHC construction, in order to validate and refine the magnetic model of the collider. Results from first attempts of the measurement and correction of nonlinear errors in the LHC experimental insertions are presented. Several discrepancies of beam-based studies with respect to the LHC magnetic model are reported.
\end{abstract}

DOI: 10.1103/PhysRevSTAB.18.121002

PACS numbers: 29.20.-c, 29.27.Bd, 41.85.-p

\section{INTRODUCTION}

Correction of nonlinear errors in low- $\beta$ insertions is expected to be of significant importance for both existing [1-5] and future colliders. In particular, operation of the Large Hadron Collider (LHC) after its high luminosity upgrade (HL-LHC) [6,7] will rely upon good quality correction of the nonlinear magnetic errors in the experimental insertions [8]. Similar limitations may also exist for SuperKEKB [9] and the Future Circular Collider (FCC) [10,11].

During its first operational run (run 1) the LHC performance was not critically limited by the presence of nonlinear errors in the experimental insertions, and no attempt was made to correct these errors in regular operation. However, upon reduction of the LHC $\beta^{*}$ from 1 to $0.6 \mathrm{~m}$ in 2012, a significant degradation of the beam lifetime was observed [12]. Optics studies at injection [13] and

*ewen.hamish.maclean@cern.ch

Published by the American Physical Society under the terms of the Creative Commons Attribution 3.0 License. Further distribution of this work must maintain attribution to the author $(s)$ and the published article's title, journal citation, and DOI. top energy [14] also revealed larger than expected discrepancies between the measured and predicted first and second order amplitude detuning. Nonlinear errors in the triplets and separation dipoles are one of several candidates for the source of these reductions in performance. Furthermore, during its second operational run the LHC will shift to operation in the more challenging $\beta^{*} \leq 0.4 \mathrm{~m}$ regime: under such conditions the compensation of these errors is expected to become a significant factor in determining the performance of the collider. Correction of nonlinear errors in experimental collider insertions is therefore a topic of both immediate relevance to the LHC, and has been and will continue to be of considerable significance in regard to the design of existing and future colliders.

Previous methods for the correction of nonlinear errors in experimental insertions, which have been employed for example at RHIC, focused on compensation of observable symptoms of the errors: either via beam-based minimization of tune shifts due to feed-down from the nonlinear errors (with linear coupling held constant) [1], or through beam-based optimization of the lifetime via scans of relevant correctors [2]. Attempts to use the former method were successful for errors of sextupolar order, however 
attempts to correct for octupole errors in the RHIC insertion regions (IRs) actually resulted in a reduction of beam lifetime and dynamic aperture [1]. In the case of the latter method, the beam lifetime is a global parameter and may not be a good figure of merit for the local corrections desired in the LHC and its successors. It should be noted that in RHIC a number of additional methods were employed to minimize the nonlinear errors in the experimental insertions during the construction phase; in particular, correction of multipole errors via shimming of the magnets based upon magnetic measurements, and selection of the highest quality magnets for use in the high luminosity insertions [15].

In contrast to the methods employed at RHIC therefore, the intended LHC and HL-LHC correction strategies are based upon the calculation of local corrections using a magnetic model of the accelerator, in principle ensuring the best possible correction quality. Two strategies have been proposed. The first method directly compensates magnetic errors in IR elements via the local minimization of selected resonance driving terms between the D1 separation dipoles left and right of the interaction point (IP) [16]. The second method is based upon a direct compensation of the IR transverse map coefficients left and right of the IP [17]. For these correction strategies to be valid however, an accurate magnetic model of the insertions is required. Magnetic measurements performed on the LHC magnets during construction provide a solid foundation for such a model, however, as will be shown in this paper, they must be both verified and refined through beam-based studies of the nonlinear dynamics.

This paper is concerned with the beam-based study of nonlinear errors in the LHC experimental insertions, and the resulting effort to simulate and correct the observed errors. The aim is not to provide a comprehensive analysis of the errors in the LHC insertions, but rather to demonstrate the validity and limitations of the selected method for beam-based study of nonlinear errors, and that the results of such measurements can be used to complement a magnetic model of a collider allowing for the determination of high quality corrections.

Section II introduces the method used to perform the beam-based measurements, wherein the method of feeddown to tune, employed at RHIC, will be extended to also consider directly measurements of the feed-down to unconstrained linear coupling. Section III will then provide details of the model to which these measurements are compared. Section IV presents the results of parasitic studies performed on IR2 which demonstrated the validity of the selected method. Section V presents the results from first dedicated studies of the IR nonlinearity performed respectively in IR1, together with results from the first attempt at local corrections of the errors in IR1. Finally, Sec. VI presents the results from studies of the nonlinear errors in IR5.

\section{BEAM-BASED STUDY OF IR NONLINEARITY}

Beam-based study of nonlinear magnetic errors can be performed by examining feed-down to readily observed beam properties. Strategies based upon feed-down to tune have previously been employed around the whole ring in SIS18 [18] and the CERN-SPS [19], and for correction of errors in the RHIC experimental insertions [1]. In the RHIC method linear coupling was held constant during the orbit bump scan. In contrast to the method employed at RHIC, in the LHC nonlinear multipoles in the IRs have been examined through their feed-down to both tune $\left(Q_{x, y}\right)$ and unconstrained linear coupling $\left(\left|C^{-}\right|\right)$. This method has a number of advantages with respect to the examination of feed-down to tune with controlled coupling: all multipoles may be examined simultaneously and the studies may be performed parasitically; however it also introduces complications: notably the possibility for large changes in linear coupling to drive shifts in the observed tune split, and the introduction of additional complexities in the simulation effort.

Table I summarizes the feed-down of normal and skew nonlinear multipoles, due to horizontal or vertical displacement from the magnetic axis, generating shifts in tune $(\Delta Q)$ and linear coupling $\left(\Delta\left|C^{-}\right|\right)$. The variation of the tune and coupling is dependent on the order of the feed-down, which in principle allows for the identification of a multipolar error from the observed change in tune and coupling upon the application of varying amplitude closed orbit bumps through the IR.

During luminosity production, the LHC operates with many bunches of particles circulating in the machine, and parasitic crossing points exist on either side of the IP. To prevent collisions at these locations, dedicated crossing angle closed orbit bumps are introduced [20]. The value of the crossing angle at the interaction point may be taken as an appropriate figure of merit to characterize the amplitude of the closed orbit bump through the insertion region; and

TABLE I. Feed-down to tune $(\Delta Q)$ and coupling $\left(\Delta\left|C^{-}\right|\right)$from nonlinear multipoles, due to horizontal or vertical displacement from the magnetic axis. In this notation $b_{3}$ is a normal sextupole, $a_{3}$ a skew sextupole.

\begin{tabular}{|c|c|c|c|c|c|c|c|}
\hline \multirow{2}{*}{$\begin{array}{l}\text { Feed-down order } \\
\text { Multipole }\end{array}$} & \multicolumn{2}{|c|}{ 1st order } & \multicolumn{2}{|c|}{2 nd order } & \multicolumn{2}{|c|}{ 3rd order } & 4 th order \\
\hline & $b_{3}$ & $a_{3}$ & $b_{4}$ & $a_{4}$ & $b_{5}$ & $a_{5}$ & $b_{6}$ \\
\hline Horizontal displacement & $\Delta Q$ & $\Delta\left|C^{-}\right|$ & $\Delta Q$ & $\Delta\left|C^{-}\right|$ & $\Delta Q$ & $\Delta\left|C^{-}\right|$ & $\Delta Q$ \\
\hline Vertical displacement & $\Delta\left|C^{-}\right|$ & $\Delta Q$ & $\Delta Q$ & $\Delta\left|C^{-}\right|$ & $\Delta\left|C^{-}\right|$ & $\Delta Q$ & $\Delta \widetilde{Q}$ \\
\hline
\end{tabular}




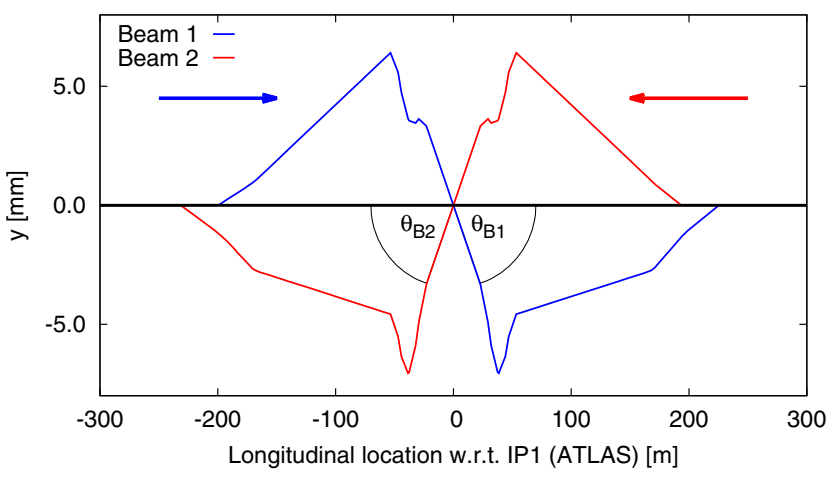

FIG. 1. Simulated nominal crossing angle in IP1 for $\beta_{\mathrm{IP} 1}^{*}=$ $0.6 \mathrm{~m}$ at $4 \mathrm{TeV}$.

during many of the studies presented in this paper it was the dedicated crossing bumps which were varied in order to examine the feed-down. Different conventions exist to describe the LHC crossing angle. Throughout the studies described in this paper the crossing angle is defined as the angle made by the beam with the longitudinal axis in its direction of travel. Figure 1 shows a simulated example of the crossing angle bump in IP1 at its nominal value of $-145 \mu \mathrm{rad}$, with the crossing angles of beam 1 and beam 2 indicated by $\theta_{B 1}$ and $\theta_{B 2}$, respectively.

Tune and linear coupling in the LHC may be measured by the so-called base-band tune (BBQ) system. The BBQ provides passive, continuous monitoring of tune and linear coupling from spectral analysis of turn-by-turn beam position data recorded at a single location per-plane-perbeam. The method by which the BBQ determines the tune is described in [21]. To measure linear coupling the BBQ utilizes a phase-locked-loop beam position monitor (BPM) arrangement and spectral analysis of turn-by-turn position data to determine the ratio between the amplitudes of the $Q_{x}$ and $Q_{y}$ components in the frequency spectra, from which the coupling coefficient may be calculated. A detailed description of the method is given in [22]. As the BBQ utilizes only turn-by-turn data from a single location, reconstruction of the frequency spectrum is necessarily limited only to position data, whereas a complete reconstruction requires the phase space data including momentum information (typically determined using BPMs separated by a $90^{\circ}$ phase advance). As a result of the partial reconstruction of the frequency spectrum it is not possible for the BBQ to distinguish sum and difference coupling (which appear as $-Q_{y}$ and $+Q_{y}$ frequencies respectively in horizontal beam spectra, and as $-Q_{x}$ and $+Q_{x}$ frequencies respectively in vertical beam spectra). In fact the BBQ measures a mixture of the sum $\left(C^{+}\right)$and difference $\left(C^{-}\right)$coupling coefficients (where $C^{+}$ and $C^{-}$characterize the magnitude of the linear coupling and are proportional to the Hamiltonian coefficients for the sum and difference resonances respectively [23,24]). In practice the linear coupling in the LHC is dominated by the difference resonance and $\left|C_{\mathrm{BBQ}}\right| \approx\left|C^{-}\right|$.
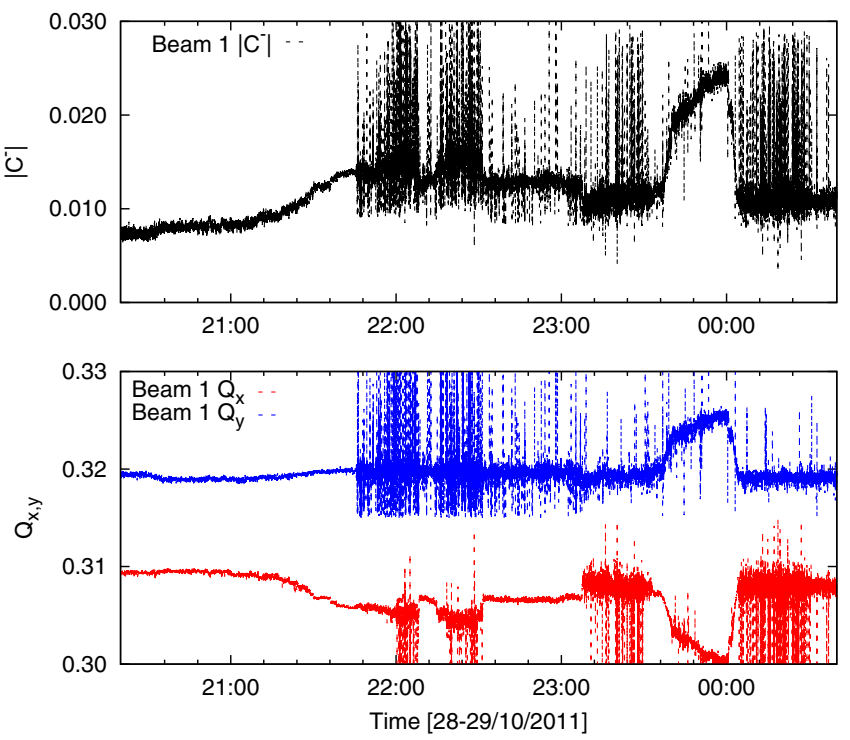

FIG. 2. An example of raw coupling (top) and tune (bottom) data recorded by the LHC BBQ for beam 1, plotted versus time.

To study the feed-down from nonlinear errors, closed orbit bumps through the IR were varied in a series of steps, pausing after each trim to collect data from the BBQ. An example of raw tune and coupling data measured by the LHC BBQ during a parasitic study of the feed-down is shown in Fig. 2 as a function of time. Where necessary, manual cleaning of the BBQ data was performed to assist with the removal of noise and misidentified spectral lines. In this procedure cuts were applied to the data within a given plateau, and the mean and standard deviation of the retained BBQ data were taken as the value and uncertainty on the tune and coupling measurements. Small transverse excitation may be applied to the LHC beams to improve the quality of the BBQ data ("chirping"). When possible, such a chirp was applied during the studies presented in this paper, however during parasitic studies it was not always possible to maintain the chirp. The significant reduction in data quality observed around 21:45 in Fig. 2 corresponded to the deactivation of the chirp midway through the measurement. In this case manual cleaning was essential in order to retrieve worthwhile data from the BBQ.

The tunes and linear coupling determined by this method were examined for dependence on the applied crossing angle trim at an IP, the observed variations providing insights into the nonlinear multipoles present in the insertion region. Correction of these field quality errors in the LHC IRs could then be performed utilizing combined $b_{3} / b_{6}$, and combined $a_{3} / b_{4} / a_{4}$ correctors (where $b_{n}$ and $a_{n}$ denote $n$th order normal and skew fields respectively, with $n=3$ indicating a sextupolar field) which are common to both beams and installed on the non-IP sides of the triplet quadrupoles left and right of the IP. A schematic of the corrector layout in the LHC experimental insertions is shown in Fig. 3. 


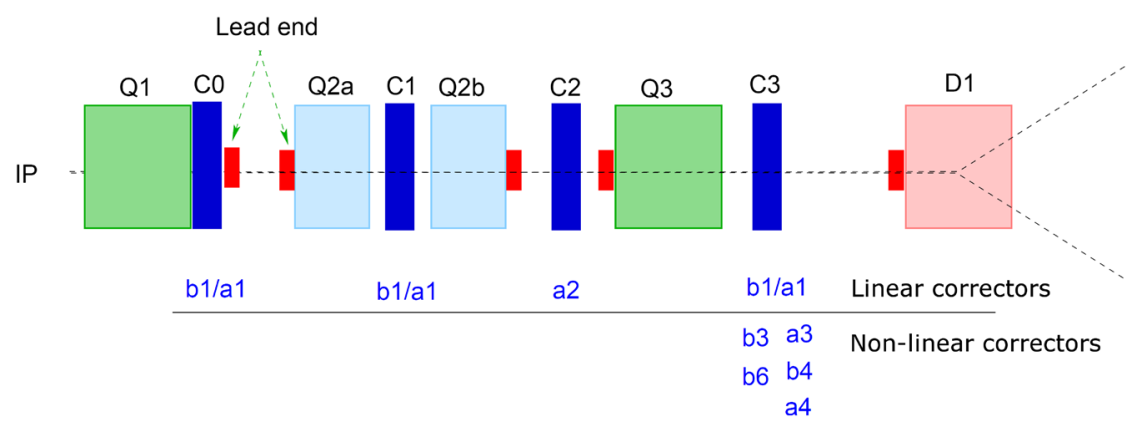

FIG. 3. Corrector layout in the LHC experimental insertions [16].

\section{SIMULATION OF THE IR NONLINEARITY}

As described in the preceding sections, the goal of the beam-based measurements is the validation and refinement of the LHC magnetic model in the IRs. To this end it was necessary to compare the observed dependence of tune and linear coupling on crossing angle with the predictions of the LHC model.

Measurements of the field quality in LHC magnets were performed for low current conditions at industry (the socalled "warm" measurements), and on a fraction of the magnets under operational conditions following their delivery to CERN (the so-called "cold" measurements). The Windows interface to simulation errors (WISE [25]) generates estimates of the magnetic errors from these measurements, and from knowledge of associated uncertainties. Uncertainties included in the WISE simulation are measurement uncertainties, power supply accuracy, hysteresis, and the uncertainty on a warm-to-cold correlation introduced for those magnets which were not measured for magnetic field quality under operational conditions. Sixty instances of the LHC errors (known as "seeds") are produced, which encompass the likely magnetic configuration of the LHC. A description of the production of the magnetic error estimates may be found in [26].

WISE estimates of magnetic errors from order $\left(b_{3}, a_{3}\right)$ to $\left(b_{11}, a_{11}\right)$ were applied to the triplet quadrupoles (Q1,Q2, Q3), the separation dipoles (D1,D2), and the matching quadrupoles (Q4-Q7), in the experimental insertions of a thin lattice MAD-X [27] model of the LHC. As the linear optics of the LHC is extremely well understood and corrected [28], with an rms beta-beat of $\sim 2 \%$, the impact of linear optics errors on the observed feed-down from nonlinear multipoles in the IR was neglected as a first approximation in these studies. The validity of this approximation will be addressed in later sections.

Closed orbit bumps, equivalent to those implemented in the real machine, were applied to this model. Predictions of the variation in tune and linear coupling with crossing angle, as determined from these simulations, could then be compared with observations. To facilitate such comparisons, the modeled tune and coupling were matched to the measured values at a given point during the closed orbit bump scan. While simple to implement in regard to the tune, the matching of the simulated linear coupling to the conditions of the real-world scan is not necessarily straightforward. Linear coupling is driven by two resonance driving terms (RDTs) [29]: $f_{1001}$ driving the difference coupling resonance, and $f_{1010}$ driving the sum coupling resonance. Of relevance to the LHC is the $f_{1001}$ RDT, approximately related to the difference coupling coefficient through Eq. (1) [29],

$$
\left|\delta Q_{\min }\right|=\left|C^{-}\right| \approx 4|\Delta|\left\langle\left|f_{1001}\right|\right\rangle
$$

where \langle\rangle indicates the average value around the ring, $\Delta$ is the unperturbed tune split (which at collision in the LHC has the nominal value of $\Delta=Q_{x \text {,unperturbed }}-Q_{y \text {,unperturbed }}=$ -0.01 ), and $\delta Q_{\min }$ is the minimum tune split defined by the coupling coefficient. Thus, under typical conditions with $Q_{x}=0.31$ and $Q_{y}=0.32$, a measured $\left\langle\left|f_{1001}\right|\right\rangle=0.05$ would correspond to $\left|C^{-}\right|=\left|\delta Q_{\min }\right|=0.002$. The relation between resonance driving terms and the coupling coefficient is discussed in more detail in [29]. The regime of validity for the approximation defined in Eq. (1) (which breaks down at the coupling resonance) is considered in [30].

The coupling measurement obtained from the LHC BBQ provides $\left|C^{-}\right|$, but does not provide details of the phase of $f_{1001}$ (which is a complex quantity). During the crossing angle scans, feed-down to a skew quadrupole introduces a coupling shift $\left(\Delta f_{1001}\right)$ which is summed with the initial $f_{1001}$ of the machine. The observed change in $\left|C^{-}\right|$depends therefore on the relative phase of $\Delta f_{1001}$ to the initial $f_{1001}$. While the magnitude of the initial $f_{1001}$ may be known, this ambiguity in the phase may result in an ambiguity of the simulated evolution of $\left|C^{-}\right|$with changing crossing angle. Ideally therefore, the coupling should be corrected close to zero, such that the initial $f_{1001}$ is negligible in comparison to that generated by feed-down. In this case the question of the RDT phase is rendered moot, and the simulated coupling can be matched to zero at the crossing angle corresponding to the observed minimum. It is in any case worth ensuring the linear coupling is well corrected prior to measurements as this also reduces the chance of a growth in 
$\left|C^{-}\right|$affecting the measured tunes. Alternatively it is possible to perform a measurement of the linear coupling independently from the BBQ by utilizing ac-dipole excitation of the beams to measure the amplitude and phase of the $f_{1001}$ around the LHC ring. This is done by performing a spectral analysis of betatron oscillations excited by an ac dipole (though this may be incompatible with the measurements being performed parasitically). A description of methods used to determine the coupling RDTs from turnby-turn BPM data of kicked beams may be found in $[24,29,31]$.

\section{DEMONSTRATION IN IR2}

LHC insertion region 2 (IR2) houses the Alice experiment, which during normal operation for proton-proton collisions does not run with tightly squeezed beams at the IP ( $\beta_{\mathrm{IP} 2}^{*}=3 \mathrm{~m}$ during $2012 \mathrm{p}$-p luminosity production). During operation for heavy ion collisions however, the beta function at the IP is significantly reduced (for example to $\beta_{\mathrm{IP2}}^{*}=0.8 \mathrm{~m}$ during $2013 \mathrm{p}-\mathrm{Pb}$ luminosity production and $\beta_{\mathrm{IP} 2}^{*}=1 \mathrm{~m}$ during the $2012 \mathrm{~Pb}-\mathrm{Pb}$ run). As a consequence, correction of the nonlinear errors in IR2 is of particular interest for heavy ion operation. To demonstrate that the nonlinear beam dynamics of the LHC experimental insertions could be examined via feed-down under the influence of varying closed orbit bumps through the IRs, as described in Secs. II and III, parasitic studies were performed in IR2 at $\beta_{\mathrm{IP} 2}^{*}=1 \mathrm{~m}$.

During commissioning of the 2011 LHC heavy-ion optics the nominal external crossing angle bump in IR2 was reversed incrementally [32]. "External" is used here to distinguish the $\pm 80 \mu \mathrm{rad}, y \leq 4 \mathrm{~mm}$, closed orbit bump through IR2, from the "internal" crossing angle bump generated by the Alice spectrometer, which is closed between the innermost triplets left and right of the IP. As the study was performed at end of fill, the large number of high intensity bunches provided tune and coupling data of a very high quality, facilitating analysis of the feed-down in IR2. Figures 4 and 5 show the modeled and measured tunes and coupling during the scan of the IR2 external crossing angle in LHC beam 1 and beam 2 respectively. Linear coupling has been matched to zero at the crossing angle corresponding to its projected minima ( $-80 \mu \mathrm{rad}$ in beam 1 and $\sim-30 \mu \mathrm{rad}$ in beam 2). Variations in the simulation between the sixty instances of the LHC defined by the WISE seeds were negligible for this configuration of the optics in IR2. The chirp was depowered during most of the scan.

Shifts to the tune and coupling are dominated by a linear variation of the $\left|C^{-}\right|$, corresponding to feed-down from $b_{3}$. The simulated variation of the coupling shows an excellent agreement with the observations, with the exception of a brief jump between -40 and $-20 \mu \mathrm{rad}$. This departure and return of the measured data to the simulation corresponded to the powering, then depowering, of the
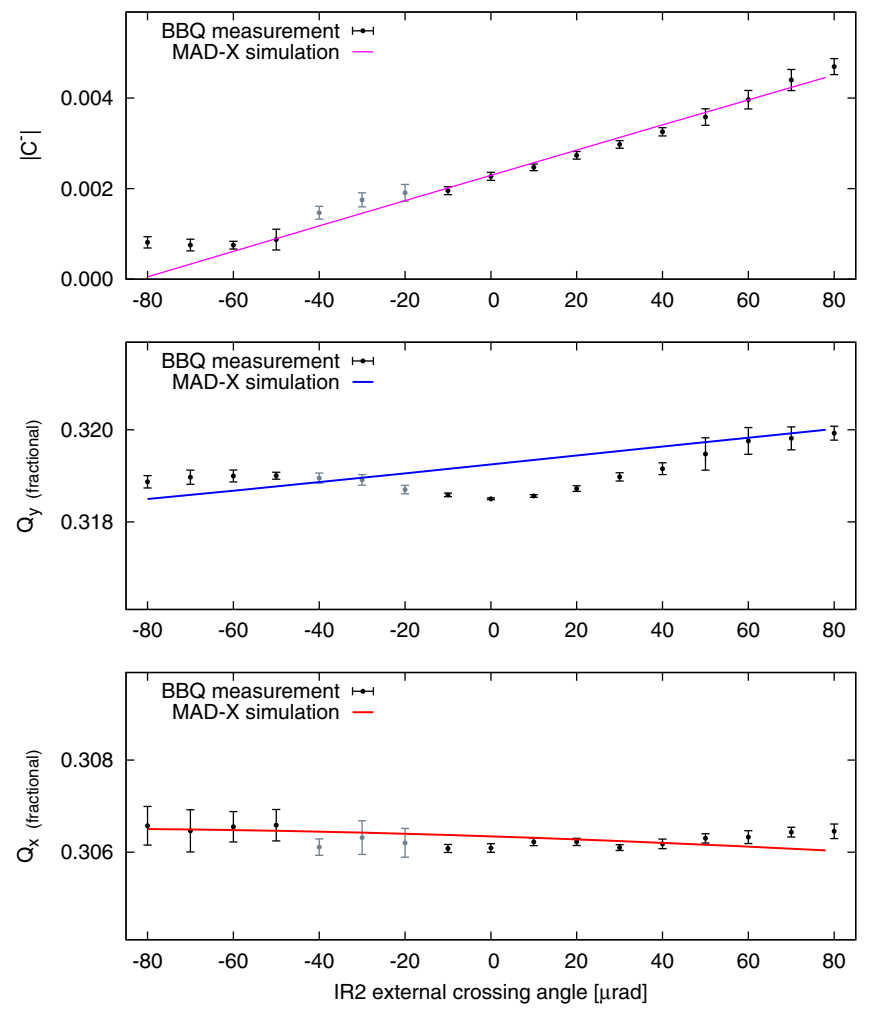

FIG. 4. Modeled and measured tunes and coupling of LHC beam 1 at $\beta_{\mathrm{IP} 2}^{*}=1 \mathrm{~m}$, plotted versus the external crossing angle. The model has been matched to produce zero coupling at the crossing angles corresponding with the projected minimum of the observed $\left|C^{-}\right|$variation. Gray points indicate data where the BBQ measurement was apparently influenced by the LHC chirp in a way which is neither normally observed in the LHC nor understood.

chirp excitation. These data points have been indicated in gray in Figs. 4 and 5. Such an influence of the chirp upon the BBQ measurement is not generally observed in the LHC, and its appearance in this data is not understood. Nonetheless the observed discrepancy at the three affected data points is not believed to significantly impact upon the conclusion that the measured and simulated coupling are in good agreement. The dominant contribution to the $b_{3}$ feed-down is identified with the D1 separation dipoles (according to the magnetic measurements, which may now be regarded as validated via the beam-based measurements). Table II details the verified $b_{3}$ components of the D1 separation dipoles at 3.5 TeV. Simulations of the crossing angle scan using thick lattice models in MAD-X and PTC indicated the contribution of the D1 fringe fields to the observed feed-down was negligible.

Variations of the tunes are significantly smaller than of the coupling (by a factor of $\sim 3$ ). In the horizontal plane the tune is roughly consistent with the predictions of the magnetic model. In the vertical plane the general trend of the tune shift is consistent with expectations from the 

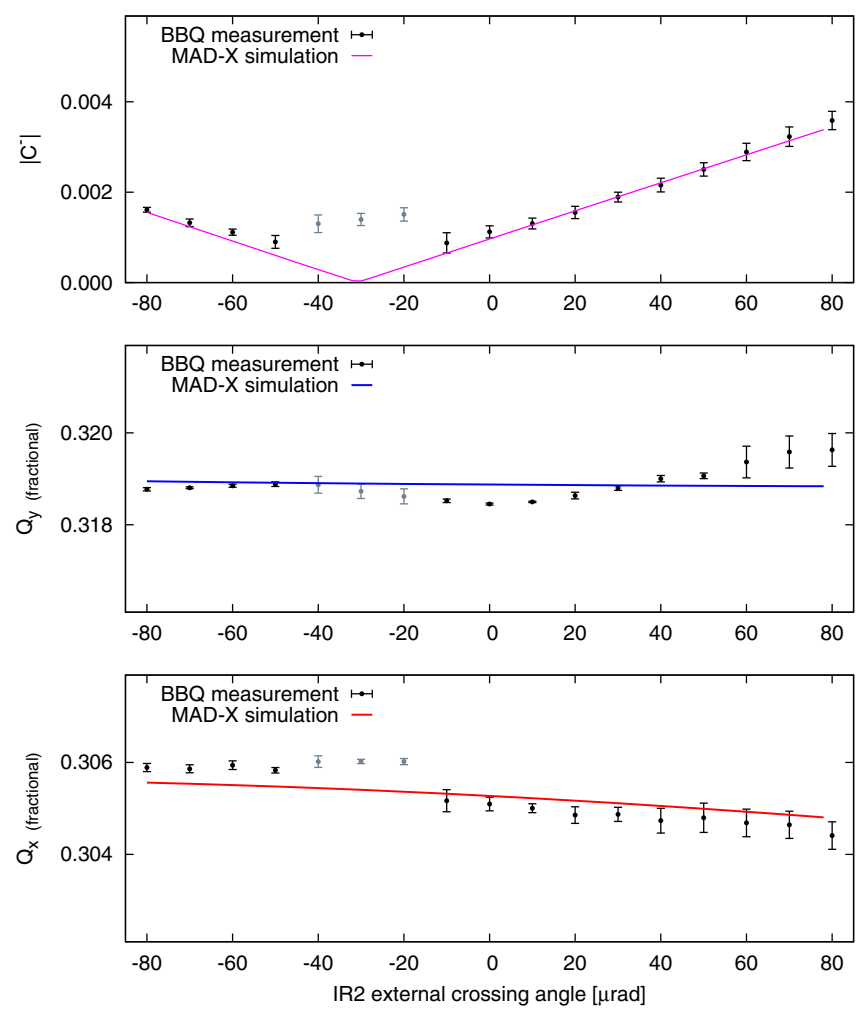

FIG. 5. Modeled and measured tunes and coupling of LHC beam 2 at $\beta_{\mathrm{IP} 2}^{*}=1 \mathrm{~m}$, plotted versus the external crossing angle. The model has been matched to produce zero coupling at the crossing angles corresponding with the projected minimum of the observed $\left|C^{-}\right|$variation. Gray points indicate data where the BBQ measurement was apparently influenced by the LHC chirp in a way which is neither normally observed in the LHC, nor understood.

model, however the measured value appears to oscillate about the prediction from simulation with an amplitude $\sim 3 \times 10^{-4}$. Such a variation is considered to be negligible, and is likely the result of drifts in the base tune.

The results above validate the use of first order feeddown to both tune and linear coupling as a method to study nonlinear errors in the LHC IRs. To demonstrate the technique for higher order multipoles however, a larger orbit excursion in the IR elements was required. Local aperture measurements during the 2011 commissioning of LHC heavy ion optics $\left(\beta_{\mathrm{IP} 2}^{*}=1 \mathrm{~m}\right)$ provided the opportunity to observe feed-down from excursions of up to

TABLE II. Verified $b_{3}$ components of the D1 separation dipoles left and right of IP2 at $3.5 \mathrm{TeV}$. The quoted multipole coefficients are values relative to the main dipole field, in units of $\left[10^{-4}\right]$, at a reference radius of $0.017 \mathrm{~m}$.

\begin{tabular}{lc}
\hline \hline Magnet & $b_{3}\left[10^{-4}\right]$ at $3.5 \mathrm{TeV}$ \\
\hline D1 left & -2.873 \\
D1 right & -0.977 \\
\hline \hline
\end{tabular}

$y \leq 25 \mathrm{~mm}$ (compared to $y \leq 4 \mathrm{~mm}$ during the spectrometer polarity reversal tests).

Unlike the measurements performed during reversal of the IR2 external crossing angle, the initial coupling of LHC during the aperture measurements was not small in comparison to the coupling generated through feed-down, and the simulated coupling could not be matched to zero. As part of the commissioning procedure however, linear optics measurements had been performed on the $\beta_{\mathrm{IP} 2}^{*}=1 \mathrm{~m}$ configuration during the LHC fill immediately prior to that used for study of the aperture. The phase of $f_{1001}$ in BPMs adjacent to the IR2 separation dipoles, measured during the linear optics studies, was therefore used as a constraint for the matching to initial conditions in the MAD-X simulation of the aperture measurement. Figure 6 presents comparisons of the $\left|C^{-}\right|$measured by the BBQ to MAD-X simulations in LHC beams 1 and 2 .

A good agreement is seen between the observations and the predictions of the magnetic model (variations between the WISE seeds were negligible) for the coupling data. This includes feed-down from multipoles of order higher than $b_{3}$, which it had not been possible to observe for smaller orbit excursions. As found during tests of the Alice spectrometer polarity reversal the feed-down is dominated by the $b_{3}$ component of the separation dipoles. Tune data collected during these measurements was of limited value. The transverse planes of the two beams were fully coupled at the start of the scan, and tune variations were in general dominated by changes of the $\delta Q_{\min }$ meaning no additional information was gained. A discrepancy between simulation and measurement was observed above $250 \mu \mathrm{rad}$ in $Q_{y}$ of beam 2 , however the large coupling makes interpretation
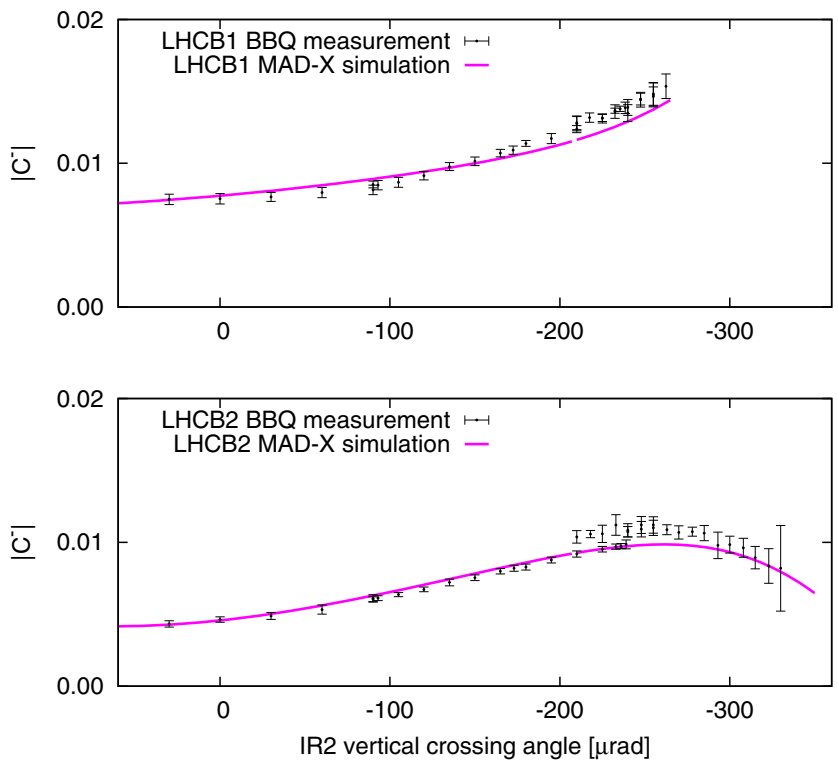

FIG. 6. Modeled and measured linear coupling of LHC beam 1 (top) and beam 2 (bottom) at $\beta_{\mathrm{IP} 2}^{*}=1 \mathrm{~m}$, plotted versus the total vertical crossing angle of IR2. 
and analysis impractical. No conclusions can be drawn for the multipoles relevant for feed-down to tune (with a vertical orbit excursion) from this data.

Parasitic studies performed during local aperture measurements in the LHC IR2, and during a test for reversal of the IR2 external crossing angle, have validated the use of first- and higher-order feed-down to linear coupling as a method to study the nonlinear errors in low- $\beta$ insertions of the LHC. This represents a useful addition to methods based solely on the tune, which have been utilized previously at other machines. In more specific terms, beam-based measurements have also verified the dominant nonlinear errors in IR2 of the LHC, allowing optimized corrections for these errors to be calculated directly from the magnetic model. The corrections at $3.5 \mathrm{TeV}$ for the resonance driving terms driven by $b_{3}$ required trims within the nominal capacity of the correctors, and in simulation were seen to compensate the feed-down to $\left|C^{-}\right|$. In practice the unavailability of one of the relevant correctors, together with a lack of available beam time, meant these corrections were not implemented during run 1 . It is expected that corrections will be included during run 2 .

\section{MEASUREMENT AND CORRECTION OF NONLINEAR ERRORS IN IR1}

Following exploratory optics measurements on the LHC at $\beta^{*}=0.4 \mathrm{~m}$ in IP1 and IP5, which house the ATLAS and CMS experiments respectively, a scan was performed of the vertical crossing angle in IR1 and feed-down to tune and linear coupling were observed in beam 2. Raw BBQ data for beam 1 during this scan was of a low quality and deemed to be unusable. The beam 2 tune and coupling observations were compared to MAD-X simulations incorporating the measured magnetic errors. Initial conditions for the simulation were matched at $0 \mu \mathrm{rad}$. Tunes and $\left|C^{-}\right|$ were matched to the values obtained from the BBQ, the phase of $f_{1001}$ was constrained to measurements obtained immediately prior to the crossing angle scan at BPMs adjacent to IP1. Figure 7 plots the measured and simulated tune and coupling shifts for the crossing angle scan. All sixty WISE seeds are plotted individually.

Feed-down to linear coupling was substantial and showed a good agreement between the model and measurement. In particular, it is possible from the available data to distinguish certain WISE seeds which offer a better agreement with the measured coupling data. The substantial variation between the seeds is due to a large uncertainty on the errors in the D1 separation dipoles. Figure 8 plots the integrated normal sextupole (top) and skew octupole (bottom) strength in the D1 dipoles left and right of the IP, with the residual $\left(\sum \frac{\left(\left|C^{-}\right|_{\text {Meas }}-\left|C^{-}\right|_{\text {Model }}\right)^{2}}{\sigma_{\text {Meas }}^{2}}\right)$ between the model predictions and the measured coupling data indicated in color. The multipole strengths $\left(K_{n}, K_{n, \text { skew }}\right)$ with dimension $\left[\mathrm{m}^{-n}\right]$ are defined by
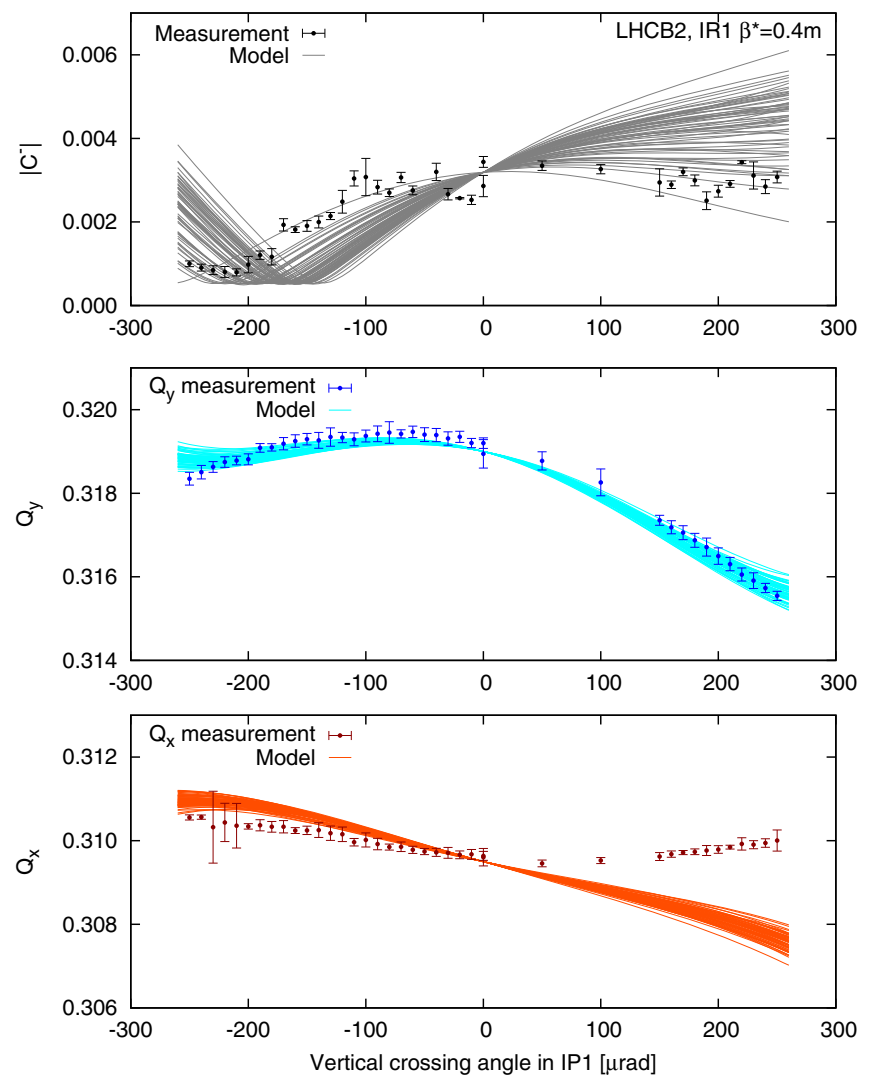

FIG. 7. Measured and modeled variation of tune and coupling in LHC beam 2, during a scan of the vertical crossing angle in IR1 at $\beta_{\mathrm{IP} 1}^{*}=0.4 \mathrm{~m}$.

$$
\begin{aligned}
B_{n}(s) & =\left.\frac{1}{(n-1) !} \frac{\partial^{n-1} B_{y}}{\partial x^{n-1}}\right|_{(0,0, s)} \\
A_{n}(s) & =\left.\frac{1}{(n-1) !} \frac{\partial^{n-1} B_{x}}{\partial x^{n-1}}\right|_{(0,0, s)} \\
K_{n} & =+\frac{q}{p}(n-1) ! B_{n} \\
K_{n, \text { skew }} & =-\frac{q}{p}(n-1) ! A_{n},
\end{aligned}
$$

The residual between model predictions and measurement depends predominantly on the $b_{3}$ component, and shows no significant trend with the skew octupole components. The best agreement to the measured data is obtained for those seeds with a small positive $b_{3}$ in the D1 separation dipole on the right side of the IP, approximately in the range $K_{3} L=0-0.25 \times 10^{-3} \mathrm{~m}^{-2}$ ). This represents a factor of 2 reduction in the uncertainty of the $b_{3}$ component of this magnet. No such obvious trend is apparent in the D1 left. With regard to Figs. 7 and 8, the true nonlinear components appear to lie towards the tails of the distribution of values generated by WISE, in such a way that the errors are smaller than would be expected from the overall wISE distribution. 

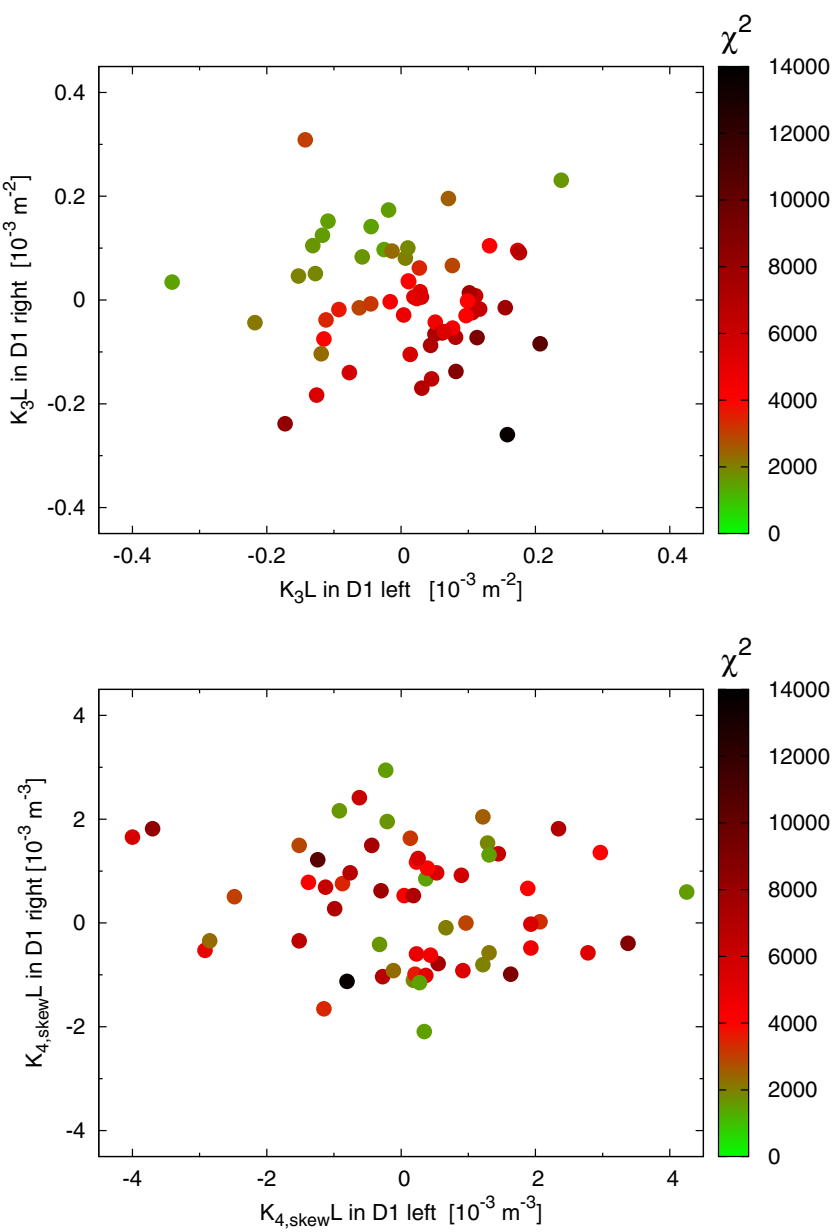

FIG. 8. Integrated normal sextupole and skew octupole components of the D1 separation dipoles left and right of IP1 at $4 \mathrm{TeV}$, for the sixty WISE seeds of the LHC. The residual $\left(\sum \frac{\left(\left|C^{-}\right|_{\text {Meas }}-\left|C^{-}\right|_{\text {Model }}\right)^{2}}{\sigma_{\text {Meas }}^{2}}\right)$ between the simulated feed-down to coupling and the measured data is indicated in color.

These results demonstrate the possibility to use measurements of feed-down to linear coupling from nonlinear errors in the LHC experimental insertions as a method to refine the magnetic model of the collider.

In regard to the tune variation, $Q_{y}$ (Fig. 7, center) shows a good agreement between measurement and simulation, however a large discrepancy is apparent in $Q_{x}$ (Fig. 7, bottom). Given this discrepancy several assumptions of the model were reexamined. BPM data were used to check orbit leakage into the LHC arcs due to nonclosure of the crossing angle bump. While the closed orbit wave generated was nonzero, the effect of the additional feed-down from the arcs was determined in simulation to be negligible $\left(\Delta Q \leq 10^{-4}\right)$. Furthermore, the excellent agreement of both first- and higher- order feed-down to $Q_{y}$ between measurement and simulation appears to constrain the real orbit bump in the machine to be approximately equal to the ideal bump applied in simulation. Analysis of the crossing angle from measured orbit data also indicated the applied closed orbit bump was approximately consistent with the expectation (measured and predicted crossing angles agreed within $30 \mu \mathrm{rad}$, which is compatible with the uncertainty due to unknown geometrical and electrical offsets of the IR BPMs). Measured transverse misalignments of the IR elements were implemented in the model and found to have a negligible impact on the predicted feed-down (several orders of magnitude below the observed tune shifts). The impact of beta-beating in the IR was also considered. No optics corrections were applied during the $\beta_{\mathrm{IP} 1}^{*}=0.4 \mathrm{~m}$ studies beyond those already implemented at $0.6 \mathrm{~m}$. Consequently as the $\beta^{*}$ was reduced, the beta-beat in IR1 increased from $\sim 2 \%$ at the nominal optics to $\leq 5 \%$ and $\leq 10 \%$ in the horizontal and vertical planes, respectively. To assess the impact of the beta-beating on the feeddown, the linear optics errors in the IR were reproduced using automatic matching routines. The software tools and methodology used, which match distortions of the propagated betatron phase, are typically intended for local correction of linear optics errors in the LHC insertions [33-36] and have been observed to give accurate descriptions of the errors [37]. Inclusion of the matched errors in the model yielded a beta-beat consistent with measurements performed via the N-BPM method [36]. The effect of this beta-beat on the simulated feed-down to tune was small $\left(\Delta Q \leq 3 \times 10^{-4}\right.$ at $\left.250 \mu \mathrm{rad}\right)$, and offered no explanation for the $Q_{x}$ observations. The source of the $Q_{x}$ discrepancy does appear therefore to be associated with a difference between the magnetic model and the real machine.

Table III shows how the $\chi_{\text {reduced }}^{2}$ of polynomial fits to the difference between the modeled and measured horizontal tune depends on which combinations of sources (equivalent to different combinations of polynomial terms) are considered. The $\chi_{\text {reduced }}^{2}$ is defined as $\chi_{\text {reduced }}^{2}=\frac{1}{\nu} \sum \frac{\left(y_{n}-f\left(x_{n}\right)\right)^{2}}{\sigma_{n}^{2}}$, where $y_{n}$ are the measured data points with error $\sigma_{n}, f\left(x_{n}\right)$ is the fitted function evaluated at the location of $y_{n}$, and $\nu$ is the number of degrees of freedom of the polynomial fit. In general a $\chi_{\text {reduced }}^{2}>1$ indicates a poor fit to the measured data, while $\chi_{\text {reduced }}^{2}<1$ may indicate an overfitting to the data.

TABLE III. $\chi_{\text {reduced }}^{2}$ of polynomial fits to $Q_{x \text {,measured }}-$ $Q_{x \text {,modeled }}$, corresponding to different combinations of possible multipole sources.

\begin{tabular}{lc}
\hline \hline Sources & $\chi_{\text {reduced }}^{2}$ \\
\hline$a_{3}+b_{4}+a_{5}+b_{6}$ & 0.14 \\
$a_{3}+b_{4}+a_{5}$ & 0.18 \\
$a_{3}+b_{4}$ & 0.39 \\
$a_{3}+b_{4}+b_{6}$ & 0.39 \\
$a_{3}+a_{5}+b_{6}$ & 0.79 \\
$b_{4}+a_{5}+b_{6}$ & 6.16 \\
\hline \hline
\end{tabular}


Contributions from $a_{3}$ (linear tune variation) are essential to achieve a good quality fit to the discrepancy, and the magnitude of the linear feed-down is approximately consistent between combinations of multipoles with a good $\chi_{\text {reduced. }}^{2}$. While it is clear therefore that there exists a skew sextupole source in the LHC missing from the magnetic model, it is not possible from the available data to distinguish between sources of order $\geq 4$. This may represent a limitation on the method if an improvement in the measurement conditions cannot be achieved.

An indication of the order of magnitude of the missing skew sextupole error can be obtained by matching the linear discrepancy between the modeled and measured $Q_{x}$ (while also constraining additional feed-down to $Q_{y}$ as zero) using the $a_{3}$ correctors left and right of IP1 (magnetic errors are included such that the initial state of the model is that of Fig. 7). The resulting corrector strengths, representing the $a_{3}$ contribution missing from the magnetic model, are given in Table IV.

From Table IV it is seen that the difference in the variation of $Q_{x}$ between model and measurement observed in Fig. 7 (bottom) represents a large discrepancy in the skew sextupole content of the LHC model in IR1 with the real machine.

Following measurements at $0.4 \mathrm{~m}$, a first attempt at correction of the nonlinear errors in IR1 was made at $\beta_{\mathrm{IP} 1}^{*}=0.6 \mathrm{~m}$. Two scans of the vertical crossing angle were performed: first with $a_{3}+b_{3}$, then $a_{3}+b_{3}+b_{4}$, corrections applied. The corrections were calculated from the magnetic model, without reference to the $0.4 \mathrm{~m}$ results, which at that stage had not yet been analyzed. As BBQ data was of a low quality and coupling data was unusable for both LHC beams, it was not possible to assess the $b_{3}$ correction quality. No attempt was made to compensate for the significant $Q_{x}$ discrepancy at $0.4 \mathrm{~m}$, described above. Figures 9 and 10 plot the measured and simulated variation of the LHC beams 1 and 2 tunes respectively throughout the crossing angle scan. Upper plots in Figs. 9 and 10 show results of the scan with $a_{3}+b_{3}$ corrections implemented, and lower plots with $a_{3}+b_{3}+b_{4}$ corrections implemented.

The $a_{3}$ correction functioned as expected in both beams, significantly compensating the linear feed-down to tune relative to predictions of the model without corrections

TABLE IV. Integrated corrector strengths required to match the linear variation of feed-down to the horizontal tune in IR1 (while leaving feed-down to $Q_{y}$ unaffected). This represents the $a_{3}$ contribution missing from the magnetic model of IR1. The integrated strength required for the matching are also given as a percentage of the corrections calculated from the magnetic measurements via minimization of the relevant RDTs.

\begin{tabular}{lcc}
\hline \hline Corrector & $K_{3, \mathrm{skew}} L\left[\mathrm{~m}^{-2}\right]$ & $\frac{\text { matched KL }}{\mathrm{RDT} \text { correction }}$ \\
\hline$a_{3}$ left of IP1 & $0.462 \times 10^{-3}$ & $38 \%$ \\
$a_{3}$ right of IP1 & $0.158 \times 10^{-3}$ & $17 \%$ \\
\hline \hline
\end{tabular}

(shown in gray). A relatively small discrepancy is observed between the measured and simulated tunes. It was found that inclusion in simulation of the same $a_{3}$ trims relative to corrections calculated from the magnetic model, detailed in Table IV, which had matched the $Q_{x}$ discrepancy at $0.4 \mathrm{~m}$, also acted to reproduce the linear tune discrepancies at $0.6 \mathrm{~m}$. This strongly implies the existence of a common source for the observed deviations between model and measurement at 0.4 and $0.6 \mathrm{~m}$, however from the data collected so far it is not possible to uniquely identify the relevant sources.

The deterioration from $\beta_{\mathrm{IP} 1}^{*}=0.6 \mathrm{~m}$ to $\beta_{\mathrm{IP} 1}^{*}=0.4 \mathrm{~m}$, of the agreement between modeled and measured feed-down from $a_{3}$, illustrates the increasing impact of nonlinear errors in the experimental insertion magnets when moving to the $\beta^{*}$ regimes relevant to the LHC's run II and beyond. Unlike the situation in IR2, these results indicate that it may not be possible to rely only upon magnetic measurements for the compensation of nonlinear errors in the LHC experimental insertions. This conclusion has notable implications for the correction strategies envisaged to date for the LHC, HLLHC and FCC, as in all these machines it has been assumed that corrections based upon magnetic measurements will be valid. Beam-based methods, either of correction or for the localization of such errors within the IR, will be required for these machines.

Application of $b_{4}$ corrections calculated from the magnetic model significantly reduced the second order tune variation. This provides some validation of the normal octupole components of the magnetic model at $\beta_{\mathrm{IP} 1}^{*}=0.6 \mathrm{~m}$. Table $\mathrm{V}$ details the applied $b_{4}$ correction in IR1.

While the octupole correction did perform well its primary function, in beam 1 implementation of the $b_{4}$ correction also generated a significant linear variation of the tune (seen in Fig. 9, bottom). Such an effect did not appear for beam 2 (Fig. 10, bottom), where the correction functioned as expected: this is in spite of the fact that in the insertion region both the errors and correctors are common to both LHC beams. That the effect appeared in one beam but not the other therefore eliminates field effects such as cross talk as a possible source of the linear tune variation. The effect must then be the result of feed-down from the correction itself, with its appearance in a single beam implying the feed-down is generated through a beam offset in the corrector, rather than by an alignment error. The scale of the offsets required to generate such a feed-down are viable (of the order of $1 \mathrm{~mm}$ ), however it is not possible to confirm this directly from BPM data due to the presence of unknown geometrical and electrical offsets in the relevant BPMs.

These observations further demonstrate the limitations of employing a correction strategy based solely upon magnetic measurements, as has been assumed will be possible for the LHC and its successors. In this case, even though the magnetic model was approximately valid, corrections 


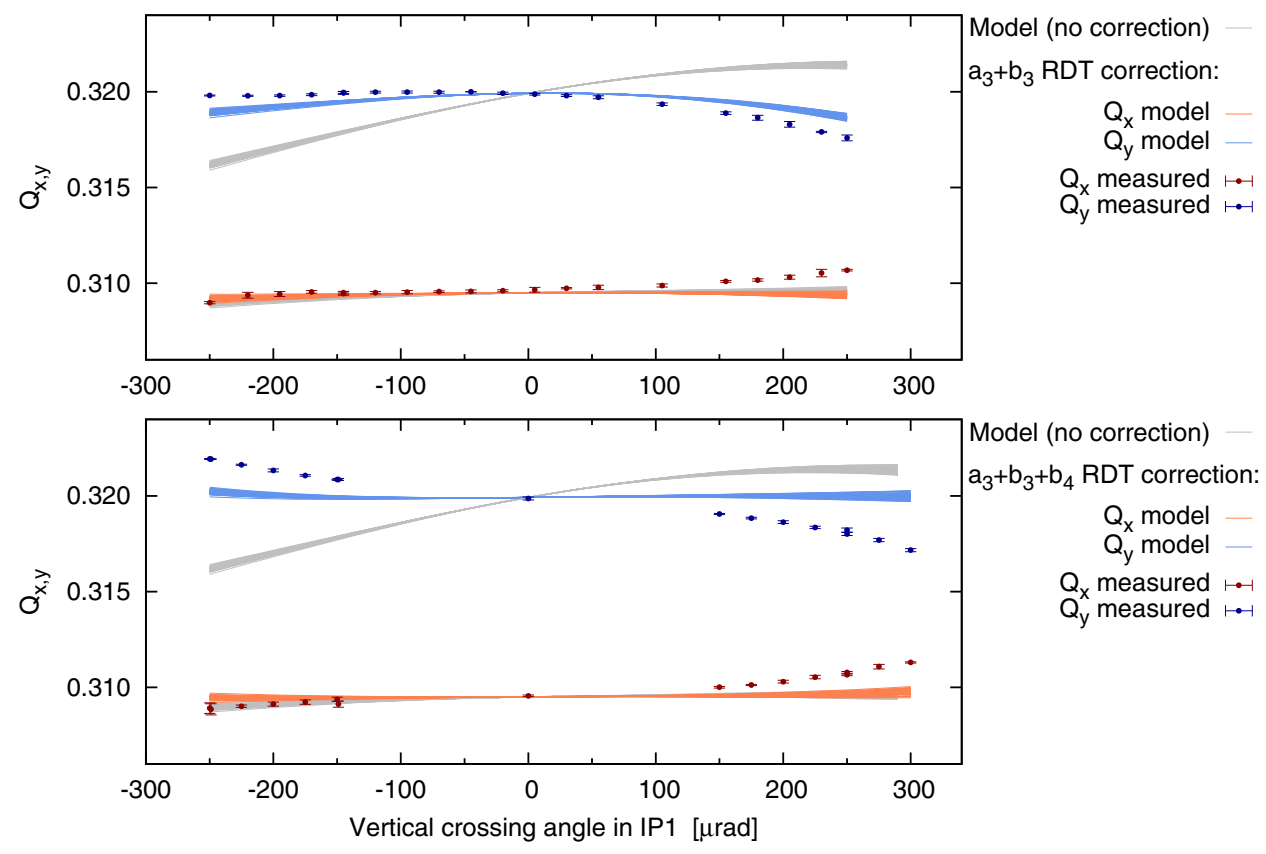

FIG. 9. Measured and modeled variation of the tunes with (vertical) IP1 crossing angle in LHC beam 1 at $\beta_{\mathrm{IP} 1}^{*}=0.6 \mathrm{~m}$. Two scans were performed: with $a_{3}$ and $b_{3}$ corrections implemented in IR1 (top) and then on addition of $b_{4}$ corrections (bottom).

based purely upon its predictions failed to correctly compensate the IR nonlinearity. If it does not prove possible to compensate this effect directly through an improved orbit then this feature of the dynamics will have to be incorporated into future correction strategies.

These results also speak directly to the beam-based methodology discussed in this paper. Observation of feed-down from the higher-order corrections demonstrates the importance of commissioning such corrections order by order. Had the $a_{3}$ and $b_{4}$ corrections been applied simultaneously, without first verifying the quality of the sextupolar compensation, the most obvious conclusion would have been failure of the $a_{3}$ correction, which may have resulted in an incorrect strategy being adopted.

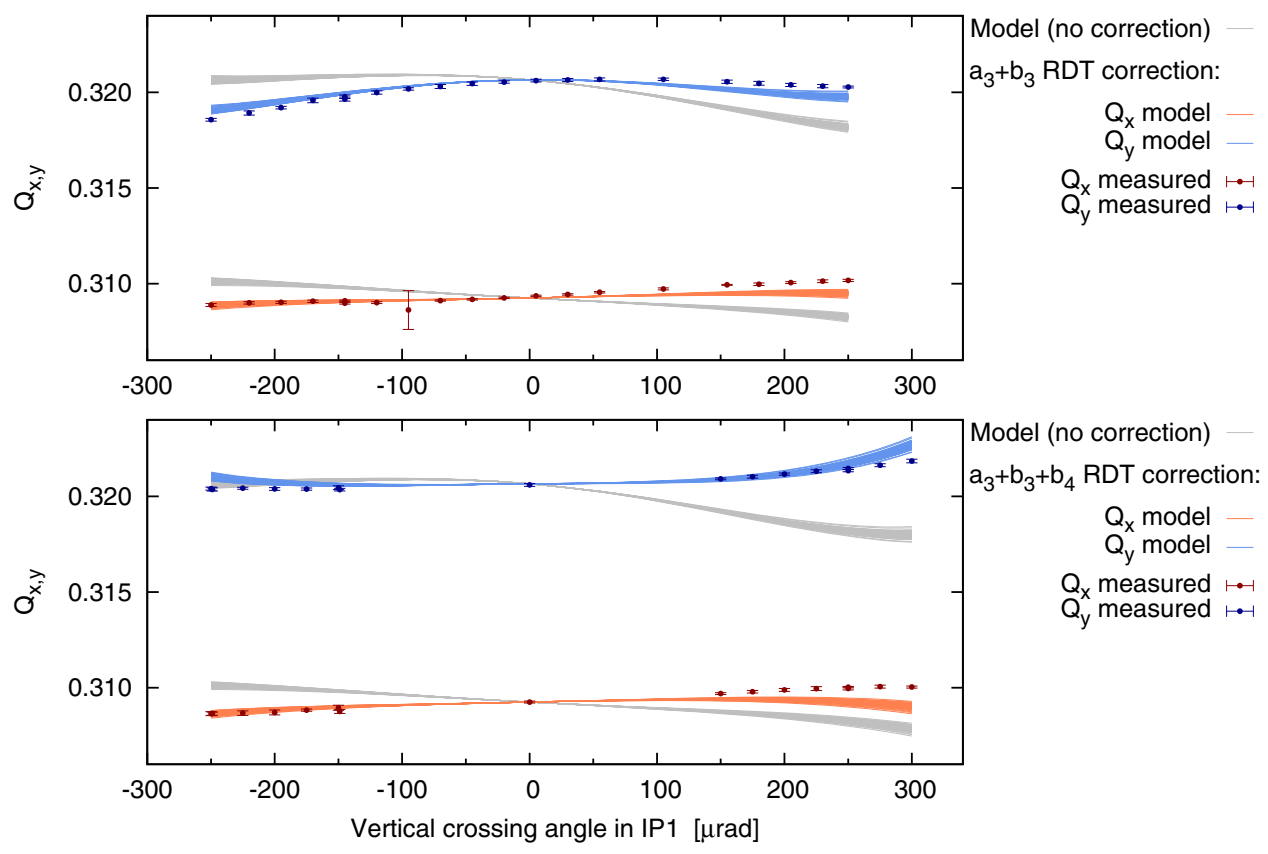

FIG. 10. Measured and modeled variation of the tunes with (vertical) IP1 crossing angle in LHC beam 2 at $\beta_{\mathrm{IP} 1}^{*}=0.6 \mathrm{~m}$. Two scans were performed: with $a_{3}$ and $b_{3}$ corrections implemented in IR1 (top) and then on addition of $b_{4}$ corrections (bottom). 
TABLE V. Applied $b_{4}$ corrections in IR1 at $\beta_{\mathrm{IP} 1}^{*}=0.6 \mathrm{~m}$, $4 \mathrm{TeV}$. The corrections shown were observed to significantly reduce the second order tune variation of both beams with vertical crossing angle in IP1.

\begin{tabular}{lr}
\hline \hline Corrector & $K_{4} L\left[\mathrm{~m}^{-3}\right]$ \\
\hline Normal octupole left of IP1 & 0.114503 \\
Normal octupole right of IP1 & -0.143734 \\
\hline \hline
\end{tabular}

\section{MEASUREMENT OF NONLINEAR ERRORS IN IR5}

Nonlinear errors in IR5 were studied at $\beta_{\mathrm{IP} 5}^{*}=0.6 \mathrm{~m}$ by scanning the horizontal crossing angle at the IP, with no corrections for the nonlinear errors in the insertion applied. Figure 11 plots the observed variation of the tune in beam 1 (top) and beam 2 (bottom), together with the predictions from simulation (BBQ data was once more of a low quality, and coupling measurements were unusable). Examination of measured closed orbit data during the scan indicated a substantial offset of the beam 2 crossing angle ( $~ 80 \mu \mathrm{rad})$ relative to the nominal bump. Measured tune data in Fig. 11 have therefore been adjusted to the crossing angles obtained from closed orbit data rather than the applied crossing angle trim.

Considerable discrepancies are observed between simulation and measurement, well outwith the uncertainty in the magnetic model. Possible deficiencies in the LHC
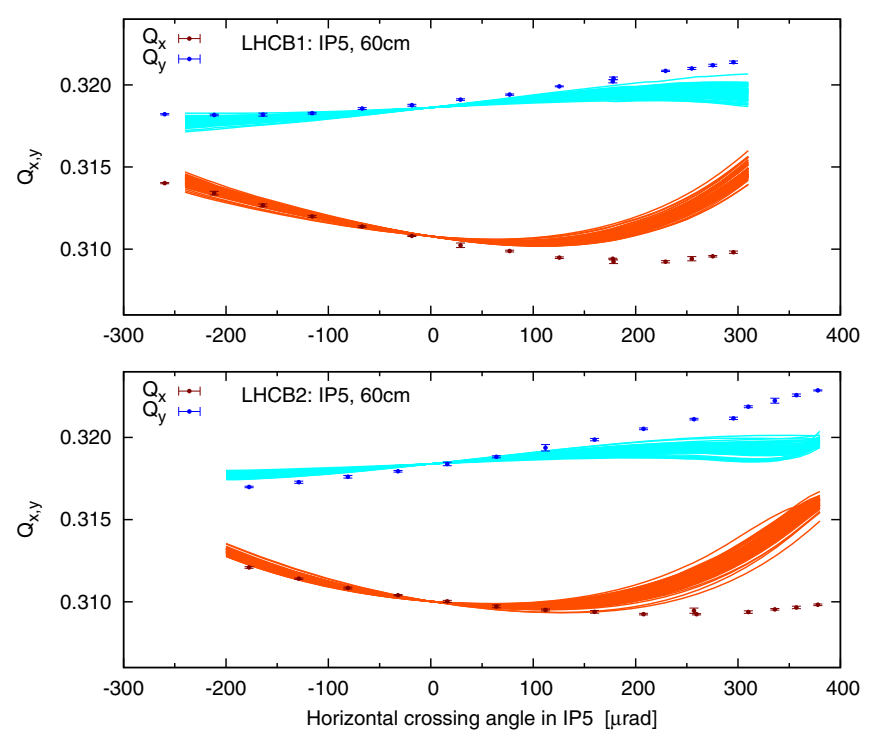

FIG. 11. Measured and modeled variation of tune in beam 1 (top) and beam 2 (bottom) with the horizontal crossing angle in IP5, at $\beta_{\text {IP5 }}^{*}=0.6 \mathrm{~m}$. Adjustments were applied to the measured tune data to reflect the crossing angle determined from measured orbit data, rather than the applied crossing angle trim. The simulation was matched to initial conditions using the measured crossing angles. model, including alignment errors in the IR, orbit leakage into the arcs, linear optics errors (which were extremely well corrected, with a beta-beat at the IP of $\sim 1 \pm 1 \%$ [36]), fringe fields, and the effect of the experimental solenoid, have been excluded as possible sources of the discrepancy in feed-down to tune (though it should be noted that the stray field of the solenoid is not currently implemented in MAD-X and it is not possible at present to comment on any effect this may have had on the tunes). This leads to the conclusion that the large discrepancies between the predicted and observed feed-down in IR5 represent an apparently quite severe discrepancy in the magnetic model. From the incomplete data obtained, however, it is not possible at this stage to identify the sources of the relevant errors. It should be noted though that the net measured errors are smaller than the predictions of the model.

These observations in IR5 clearly demonstrate that in order to correct for nonlinear errors in the LHC experimental insertions it is insufficient to rely purely upon magnetic measurements performed during construction. This undermines the correction strategies which have been so far assumed for the LHC, HL-LHC and FCC, and some form of beam-based study and correction of IR nonlinearities will therefore be of significant importance for the operation of these machines.

\section{CONCLUSIONS}

Local correction of nonlinear errors in experimental insertions is of immediate concern in regard to the LHC's second run, and is also a significant concern in regard to the design of the HL-LHC [7] and future colliders such as the FCC [11]. The optimal correction strategies intended for these colliders are based on either the minimization of selected resonance driving terms through the IR, or the direct compensation of transverse map components either side of the IP. Both techniques represent breaks with previous practices, which have utilized beambased compensation of observable symptoms of the nonlinear errors, and instead require a magnetic model of the insertion from which to calculate corrections. Magnetic measurements performed during construction of a collider can provide such a magnetic model.

Previous methods to correct nonlinear errors in experimental insertions at RHIC have utilized feed-down to tune, with linear coupling held constant. Results, presented in this paper, of parasitic measurements performed on the LHC's IR2 have demonstrated that, in addition to feed-down to tune, it is also possible in the LHC to study such nonlinear errors via their first- and higher-order feed-down to linear coupling. Such coupling measurements were used to verify magnetic measurements of the dominant errors in IR2, namely the $b_{3}$ component of the D1 separation dipoles. Furthermore, studies of the nonlinear errors in IR1 at $\beta_{\mathrm{IP} 1}^{*}=0.4 \mathrm{~m}$ demonstrated that it was possible to use such beam-based 
methods to refine the magnetic model of the LHC. In particular the uncertainty in the normal sextupole component of the D1 dipole right of IP1 was reduced by a factor of 2 via analysis of the feed-down to coupling.

Observations of feed-down to tune in the LHC's IR1 and IR5 however, revealed the existence of large discrepancies between the magnetic model and the real machine. In IR1 the source was identified as a significant discrepancy between the modeled and measured skew sextupole content of the IR, together with higher order discrepancies that could not be distinguished. It was not possible from the available data to localize the sources within the IR. In IR5 neither the order nor location of the errors could be determined from the incomplete data collected during run I. The existence of such large discrepancies between the predictions of the magnetic model and observations of the real machine undermine the correction strategies intended for the LHC and its successors, which have assumed the validity of a magnetic model based upon magnetic measurements performed during construction. While some progress has been achieved, therefore, in the validation and refinement of the LHC's magnetic model using beam-based methods, significant challenges remain if an optimized performance of the LHC is to be obtained in the $\beta^{*}$ regime relevant to run II and beyond. Further application and development of beam-based techniques for the study of these errors will therefore be a priority during the LHC's second run.

\section{ACKNOWLEDGMENTS}

Particular thanks go to Stefano Redaelli and the LHC aperture measurement team for allowing parasitic observations during IR 2 aperture measurements, and similarly to John Jowett for allowing tests of the IR2 crossing angle reversal to be used for these studies together with assistance he rendered in regard to the reproduction of the Alice internal and external crossing angles in MAD-X. Thanks also go to Gabriel Müller for assisting in the extraction of data. None of these measurements would have been possible without the members of the CERN Optics Measurement and Correction team, past and present, who have contributed to the optics tools, measurements, and corrections which were utilized in these studies. Finally thanks go to all the Engineers In Charge and operations crew who assisted with the measurements described above. One of the authors (E. H. M.) acknowledges financial support for these studies provided in the initial stages by CERN and the John Adams Institute for Accelerator science (University of Oxford), then later by the Cockcroft Institute and University of Manchester through the HL-LHC Work Package 2. The support of Hertford College (University of Oxford) is also acknowledged. The HiLumi LHC Design Study (a subsystem of HL-LHC) is cofunded by the European Commission within the Framework Program 7 Capacities Specific Program, Grant Agreement No. 284404.
[1] F. Pilat, Y. Luo, N. Malitsky, and V. Ptitsyn, Beam-based nonlinear optics corrections in colliders, in Proceedings of the 21st Particle Accelerator Conference, Knoxville, TN, 2005 (IEEE, Piscataway, NJ, 2005).

[2] W. Fischer, J. Beebe-Wang, Y. Luo, S. Nemesure, and L. Rajulapati, RHIC proton beam lifetime increase with 1 0- and 12-pole correctors, in Proceedings of the first International Particle Accelerator Conference, Kyoto, Japan (IPAC'10/ACFA, Kyoto, 2011), THPE099.

[3] H. Grote, F. Schmidt, and L.H. A. Leunissen, LHC dynamic aperture at collision, Technical Report No. LHCPROJECT-NOTE-197, 1999 [https://cds.cern.ch/record/ 691988?n].

[4] O. S. Brüning, S. Fartoukh, and M. Giovannozz, Field quality issues for LHC magnets: Analysis and perspectives for quadrupoles and separation dipoles, Technical Report No. CERN-AB-2004-014-ADM [https://cds.cern.ch/ record/712881].

[5] L. C. Teng, Error analysis for the low beta quadrupoles of the Tevatron collider, Technical Report No. FERMILABTM-1097 [http://inspirehep.net/record/177853?ln].

[6] L. Rossi, LHC upgrade plans: Options and strategy, in Proceedings of the 2nd International Particle Accelerator Conference, San Sebastián, Spain (EPS-AG, Spain, 2011), TUYA02.

[7] HiLumi LHC Collaboration, HL-LHC preliminary design report: Deliverable: D1.5, Technical Report No. CERNACC-2014-0300 [https://cds.cern.ch/record/1972604?ln].

[8] M. Giovannozzi, S. Fartoukh, and R. de Maria, Triplet correctors specifications, The 3rd joint HiLumi LHC-LARP annual meeting [http://cds.cern.ch/record/1954722?ln].

[9] H. Sugimoto, Superkekb, The CERN-ICFA workshop on advanced optics control, 2015 [https://indico.cern.ch/ event/349643/overview].

[10] Daniel Schulte, Optics challenges for future hadron colliders, The CERN-ICFA workshop on advanced optics control, 2015 [https://indico.cern.ch/event/349643/overview].

[11] M. Benedikt, D. Schulte, J. Wenninger, and F. Zimmerman, Challenges for highest energy circular colliders, Technical Report No. CERN-ACC-2014-0153 [https://cds.cern.ch/ record/1742294?ln].

[12] G. Arduini, Closing discussion ("AOBs and wrap up"), The LHC Studies Working Group day, 2014 [http://indico.cern .ch/event/331020/].

[13] E. H. Maclean, R. Tomás, F. Schmidt, and T. H. B. Persson, Measurement of LHC nonlinear observables using kicked beams, Phys. Rev. ST Accel. Beams 17, 081002 (2014).

[14] S. White, E. Maclean, and R. Tomás, Direct amplitude detuning measurement with ac dipole, Phys. Rev. ST Accel. Beams 16, 071002 (2013).

[15] J. Wei and M. Harrison, The RHIC project—design, status, challenges, and perspectives. in multi-GeV high-performance accelerators and related technology, in Proceedings of the 16th RCNP International Symposium, Osaka, Japan, 1997 (World Scientific, Singapore, 1997), No. C97-0312.1, pp. 198-206 [http://www.rhichome.bnl.gov/AP/ ap_notes/RHIC_AP_123.ps].

[16] O. Bruning, S. Fartoukh, M. Giovannozzi, and T. Risselada, Dynamic aperture studies for the LHC separation 
dipoles, LHC Project Note 349 [https://cds.cern.ch/record/ 742967? ln].

[17] R. Tomás, M. Giovannozzi, and R. de Maria, Nonlinear correction schemes for the phase 1 LHC insertion region upgrade and dynamic aperture studies, Phys. Rev. ST Accel. Beams 12, 011002 (2009).

[18] G. Franchetti, A. Parfenova, and I. Hofmann, Measuring localized nonlinear components in a circular accelerator with a nonlinear tune response matrix, Phys. Rev. ST Accel. Beams 11, 094001 (2008).

[19] A. Parfenova, G. Franchetti, R. Tomás, and G. Vanbavinckhove, Benchmarking of the ntrm method on octupolar nonlinear components at the cern-sps synchrotron, in Proceedings of IPAC 2010 (IPAC'10/ACFA, Kyoto, 2010), WEOBRA01.

[20] LHC design report v.1: The LHC Main Ring, edited by O. Brüning et al. CERN, 2004 [https://cds.cern.ch/record/ 782076?ln]

[21] M. Gasior and R. Jones, The principle and first results of betatron tune measurement by direct diode detection, Technical Report No. LHC-Project-Report 853 [http:// cds.cern.ch/record/883298?ln].

[22] R. Jones, P. Cameron, and Y. Luo, Towards a robust phase locked loop tune feedback system, Technical Report No. C-A/AP/\#204 [http://cds.cern.ch/record/895167?ln].

[23] G. Guignard, Betatron coupling and related impact of radiation, Phys. Rev. E 51, 6104 (1995).

[24] R. Calaga, R. Tomás, and A. Franchi, Betatron coupling: Merging Hamiltonian and matrix approaches, Phys. Rev. ST Accel. Beams 8, 034001 (2005).

[25] Wise homepage, http://wise.web.cern.ch/WISE/.

[26] P. Hagen, WISE- user guide and implementation notes, [http://wise.web.cern.ch/WISE/Doc/lhc-project-report-wise .pdf].

[27] MAD—methodical accelerator design, http://mad.web.cern .ch $/ \mathrm{mad} /$.

[28] R. Tomás, R. Calaga, A. Langner, Y. I. Levinsen, E. H. Maclean, T. H. B. Persson, P. K. Skowronski, M. Stzelczyk, G. Vanbavinckhove, and R. Miyamoto, Record low $\beta$-beating in the LHC, Phys. Rev. ST Accel. Beams 15, 091001 (2012).
[29] A. Franchi, Studies and measurements of linear coupling and nonlinearities in hadron circular accelerators, Ph.D. thesis, Universität Frankfurt, 2006.

[30] T. Persson and R. Tomás, Improved control of the betatron coupling in the Large Hadron Collider, Phys. Rev. ST Accel. Beams 17, 051004 (2014).

[31] R. Miyamoto, R. Calaga, M. Aiba, R. Tomás, and G. Vanbavinckhove, Measurement of coupling resonance driving terms in the LHC with ac dipoles, Technical Report No. CERN-ATS-2011-158 [https://cds.cern.ch/record/ 1382073/files/?n].

[32] R. Alemany-Fernandez, G. H. Hemelsoet, J. M. Jowett, M. Lamont, D. Manglunki, S. Redaelli, M. Schaumann, R. Versteegen, and J. Wenninger, Alice spectrometer polarity reversal, Technical Report No. ATS/Note/2012/039 MD [https://cds.cern.ch/record/1441694?ln].

[33] R. Tomás, O. Bruning, M. Giovannozzi, P. Hagen, M. Lamont, F. Schmidt, G. Vanbavinckhove, M. Aiba, R. Calaga, and R. Miyamoto, CERN Large Hadron Collider optics model, measurements, and corrections, Phys. Rev. ST Accel. Beams 13, 121004 (2010).

[34] G. Vanbavinckhove, Optics measurements and corrections for colliders and other storage rings, Ph.D. thesis, Universiteit van Amsterdam, 2012.

[35] R. Tomás, T. Bach, J. Coello, V. Kain, M. Kuhn, A. S. Langner, Y. I. Levinsen, K. S. B. Li, E. H. Maclean, V. Maier, N. Magnin, M. J. McAteer, T. H. B. Persson, P. Skowronski, R. Westenberger, and S. White, Prospects for the LHC optics measurements and corrections at higher energy, in Proceedings of the 5th International Particle Accelerator Conference, Dresden, 2014 (JACoW, Geneva, 2014), TUPRO018.

[36] A. Langner and R. Tomás, Optics measurement algorithms and error analysis for the proton energy frontier, Phys. Rev. ST Accel. Beams 18, 031002 (2015).

[37] T. Bach, P. Hagen, A. Langner, Y. I. Levinsen, M. McAteer, E. H. Maclean, T. Persson, P. Skowronski, R. Tomás, E. Todesco, and S. White, MQY $1 \%$ calibration errors, Technical Report No. CERN-ATS-Note-2013-029 MD [https://cds.cern.ch/record/1545888/files/?ln]. 